\title{
Transthoracic robotic first rib resection: Twelve steps
}

\author{
Bryan M. Burt, MD, ${ }^{\mathrm{a}}$ Nihanth Palivela, BS, ${ }^{\mathrm{a}}$ Anahita Karimian, BSN, RN, CNOR, ${ }^{\mathrm{b}}$ and
} Michael B. Goodman, CST, ${ }^{\mathrm{b}}$ Houston, Tex

\footnotetext{
From the a'Division of Thoracic Surgery, Michael E. DeBakey Department of Surgery, Baylor College of Medicine, Houston, Tex; and b Baylor St Luke's Medical Center, Houston, Tex.

Disclosures: Authors have nothing to disclose with regard to commercial support.

Received for publication Nov 18, 2019; revisions received Nov 18, 2019; accepted for publication Jan 3, 2020; available ahead of print Feb 10, 2020.

Address for reprints: Bryan M. Burt, MD, Division of Thoracic Surgery, Michael E. DeBakey Department of Surgery, Baylor College of Medicine, One Baylor Plaza, Houston, TX 77030 (E-mail: Bryan.Burt@bcm.edu).

JTCVS Techniques 2020;1:104-9

2666-2507

Copyright $\subset 2020$ The Author(s). Published by Elsevier Inc. on behalf of The American Association for Thoracic Surgery. This is an open access article under the CC BY-NC-ND license (http://creativecommons.org/licenses/bync-nd/4.0/).

https://doi.org/10.1016/j.xjtc.2020.01.005
}

$\nabla$ Video clip is available online.

Thoracic outlet syndrome (TOS) is a trio of debilitating musculoskeletal disorders that result from compression of the neurovascular structures that serve the upper extremity, common types of TOS are neurogenic TOS in which compression of the brachial plexus results in disabling upper-extremity pain and paresthesias, and venous TOS (vTOS), which results in subclavian vein thrombosis, upper-extremity swelling, and cyanosis secondary to subclavian vein compression. ${ }^{1}$ TOS is treated surgically by first rib resection (FRR), the traditional surgical approaches of which are supraclavicular and transaxillary. An infraclavicular approach, either alone or in combination with a supraclavicular approach (paraclavicular), is often used in cases of vTOS in which it is important to resect the anterior most aspect of the first rib. Each of these approaches has at least some limitation for achieving optimal exposure of the first rib, and this is commonly amplified in patients with obese body habitus or muscular stature. Each of these approaches requires at least some degree of retraction or manipulation of central neurovascular structures that include the brachial plexus, the phrenic nerve, and the subclavian artery and vein. The emerging transthoracic robotic approach to FRR provides striking exposure of the near entirety of the first rib without any retraction. It is described in 12 steps.

\section{MATERIALS AND METHODS}

The technique of transthoracic robotic FRR (rFRR) is described in a series of distinct 12 steps, each with an accompanying video. The described approach uses the da Vinci Xi system (Intuitive Surgical, Sunnyvale, Calif) and a Midas Rex handheld surgical drill (Medtronic, Minneapolis, Minn). Full instrumentation is provided in the case card that is included as Figure 1. each defined by a distinct clinical presentation. The most

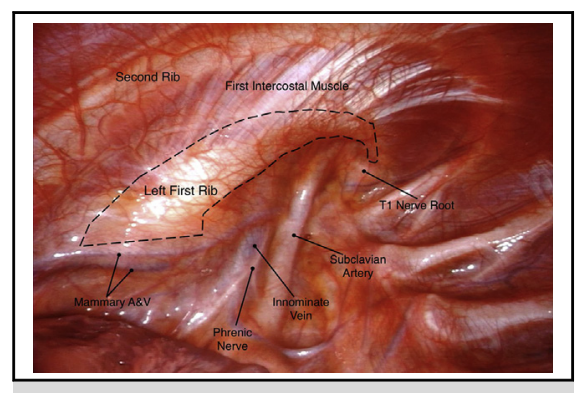

Unrivaled exposure of the first rib by a transtho-

\section{CENTRAL MESSAGE \\ The transthoracic robotic approach to FRR offers unparal- leled exposure of the first rib and is anticipated to minimize oper- ative morbidity and favorably disrupt the field of TOS.}

See Commentaries on pages 110 and 112. racic robotic approach.

\section{RESULTS}

The rFRR technique is demonstrated in an 18-year-old female athlete with left Paget-Schroetter syndrome 4 weeks after successful catheter-based lysis of a subclavian vein thrombosis. Single lung isolation is achieved with a double-lumen tube or bronchial blocker, and the patient is placed in the full lateral decubitus position. An arterial line and urinary catheter are considered optional. The procedure is distilled into 12 steps:

1. Port Placement (Video 1): Three 8-mm ports and one 10-mm assistant port are used. The border of the scapula is marked. The site of the posterior port is marked just inferior to or slightly posterior to the tip of the scapula. The site of the anterior port is marked at approximately the fourth interspace, just anterior to the anterior axillary line. Equidistant from and slightly inferior to these 2 working ports, the camera port is marked, generally corresponding to approximately the seventh interspace in the mid axillary line. The 8-mm camera port incision is made, and the chest is entered with a clamp. A port is placed, the camera is inserted, and insufflation is begun at $8 \mathrm{~mm}$ of pressure 


\section{Burt Robotic FRR}

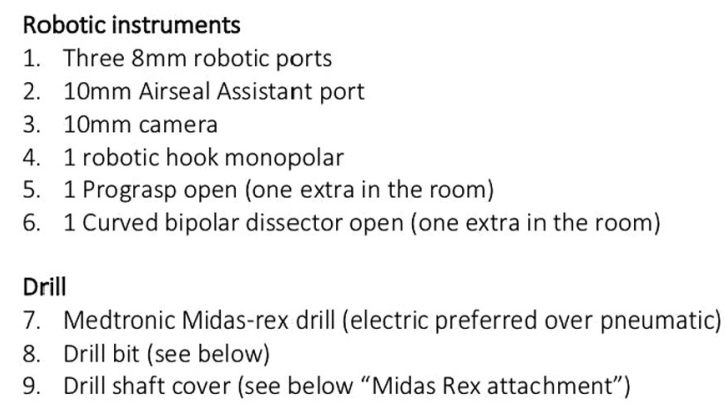

Other

10. Double action ring foreceps

11. Laparoscopic grasper

12. Suction Irrigator

13. Cigar sponges

14. Long (10 inch) $4 \mathrm{~mm}$ and $5 \mathrm{~mm}$ Kerrison Rongeurs

15. Surgicel
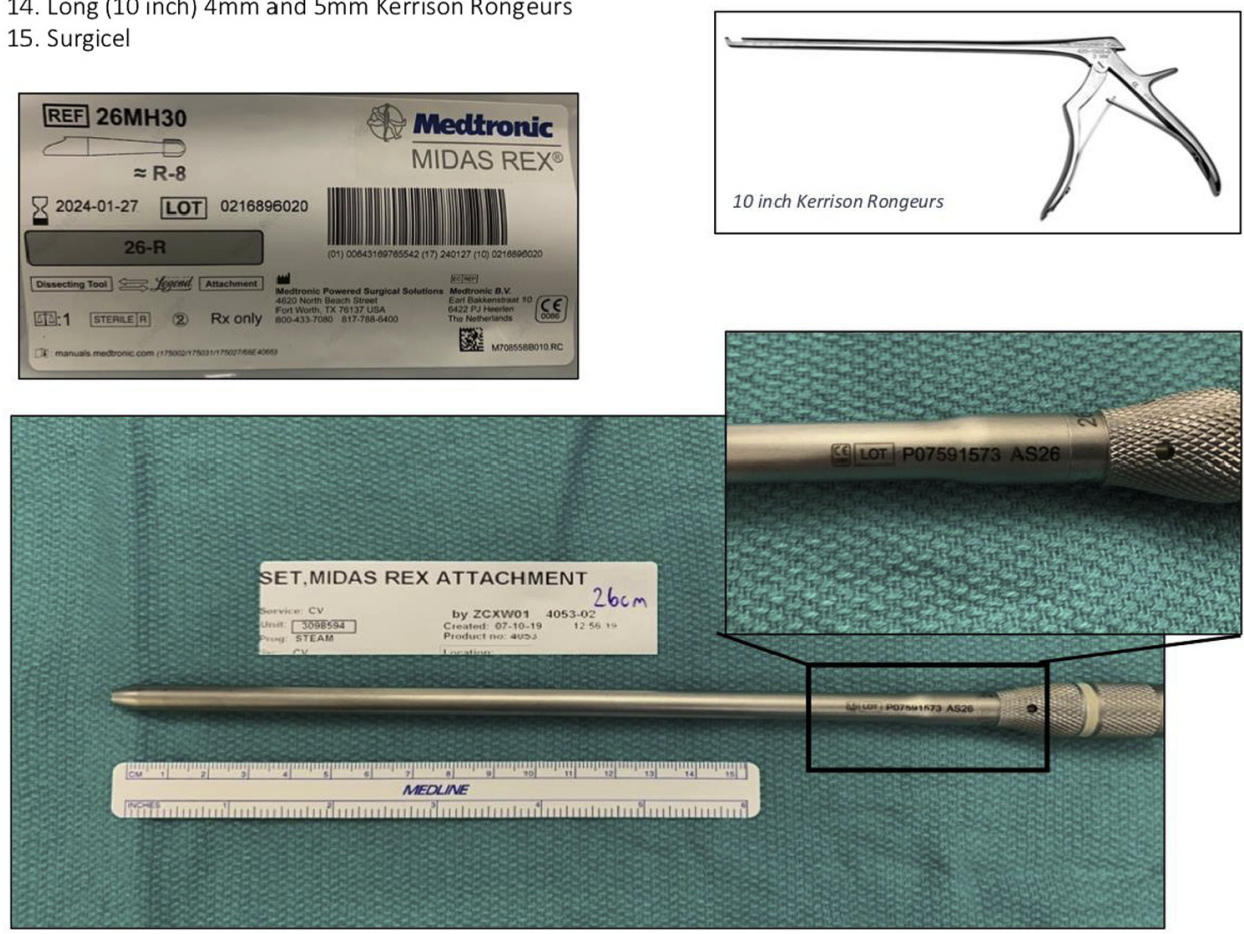

FIGURE 1. Burt rFRR.

once safe pleural entry is confirmed. We used a 0degree camera; however, a 30-degree camera can also be used. Intercostal nerve blocks are placed with $3 \mathrm{~mL}$ of dilute Marcaine placed in the fourth to ninth intercostal spaces posteriorly. The posterior port is placed in the fifth of sixth intercostal space, and the anterior port is placed in the fourth (but sometimes third or fifth) intercostal space. The position of the working ports is critical to properly accommodate the vector of the handheld surgical drill later in the case. The surgeon may want to pilot the drill's trajectory by placing a laparoscopic instrument through the posterior port to ensure an efficient route to both the posterior and anterior aspects of the rib. On occasion, drilling can occur from the anterior port, so the same can be done for the anterior port. An assistant port is placed anteriorly and inferiorly in a convenient location, typically around the ninth interspace in the anterior axillary line.

2. Survey of Anatomy (Video 2). A survey of the magnificent anatomy of the thoracic outlet is performed, illustrated in Video 2. The presence of an accessory phrenic 


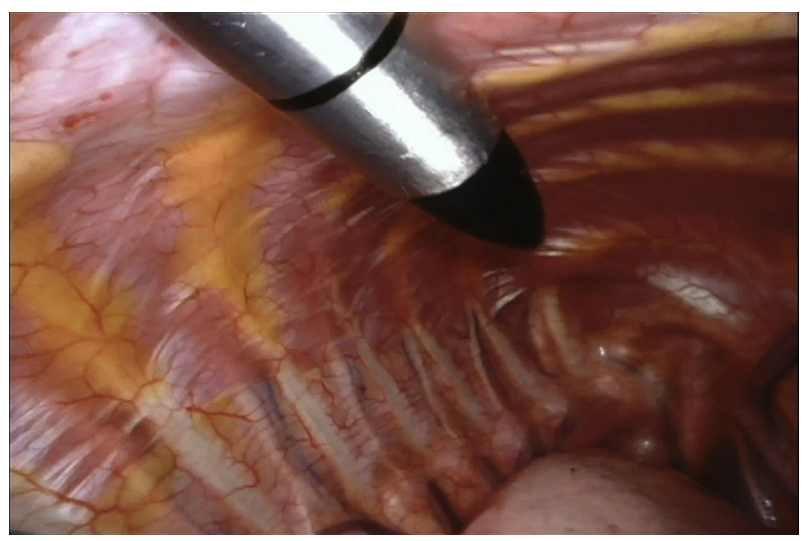

VIDEO 1. Port placement. Video available at: https://www.jtcvs.org/ article/S2666-2507(20)30017-1/fulltext.

nerve is not uncommon, and cadaveric studies have demonstrated its presence within the thorax of in $41 \%$ of cadavers.

3. Separation of First Intercostal Muscle From First Rib (Video 3). A robotic hook monopolar cautery instrument (setting 4) is used to incise the parietal pleura and to separate the first intercostal muscle from its attachments to the lateral aspect of the first rib from the level of the thoracic artery and vein anteriorly to the most posterior aspect of the first rib.

4. Dissection of Mediastinal Pleura From Inferior Aspect of First Rib (Video 4). The mediastinal pleura is dissected from the inferior aspect of the first rib using blunt dissection and sparing energy. Although this step is not critical, it defines the medial border of the first rib and opens the space between the rib and the neurovascular contents of the thoracic outlet, which prepares the rib for safe division.

5. Division of Costal Cartilage and Preparation of Posterior Rib (Video 5). The transthoracic approach pro-

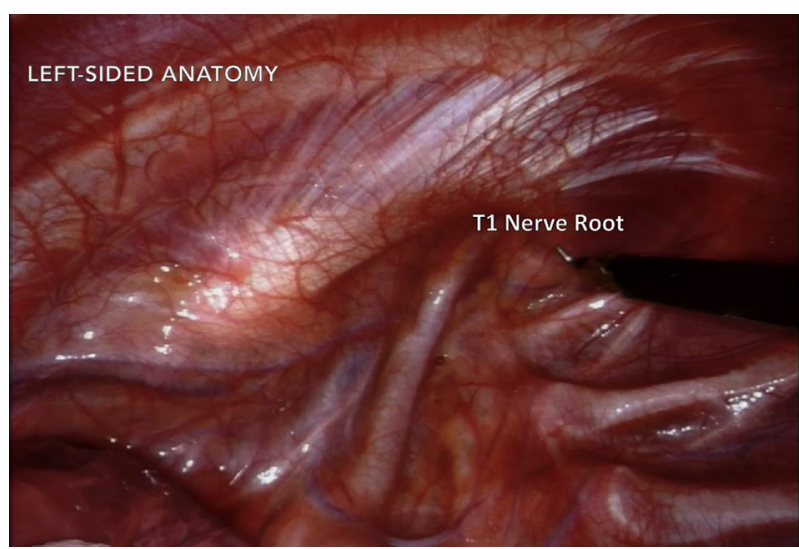

VIDEO 2. Survey of anatomy. Video available at: https://www.jtcvs.org/ article/S2666-2507(20)30017-1/fulltext.

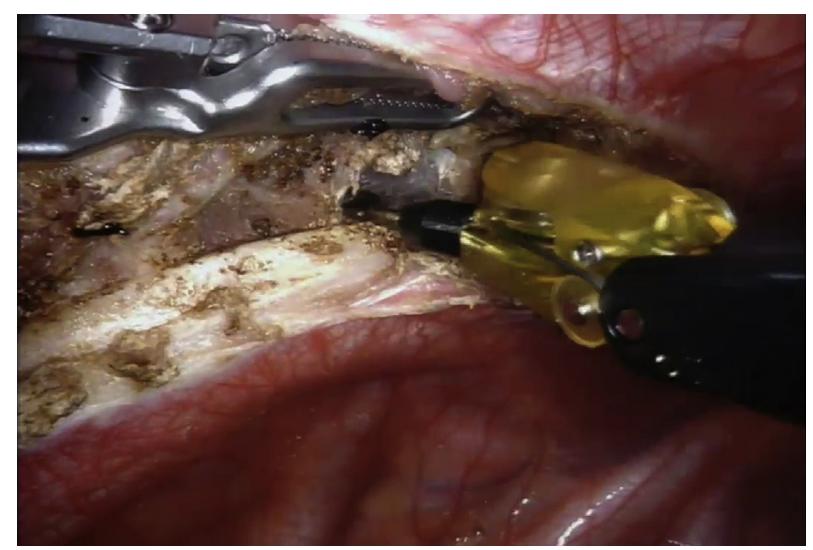

VIDEO 3. Separation of first intercostal muscle from first rib. Video available at: https://www.jtcvs.org/article/S2666-2507(20)30017-1/fulltext.

vides wide exposure of the anterior first rib its cartilage, which can often be simply divided with monopolar (or bipolar) energy. The surgeon will appreciate active suction of the resultant opaque smoke by the bedside assistant during cartilage division. In cases in which cartilage is partially (or completely) ossified, its bony portion can be divided later with the drill. Dissection of the posterior first rib is then performed to prepare a clean line for its division. A curved bipolar dissector is used for this portion of the case and the remainder of the procedure (setting 6) as the T1 nerve root is relatively approximate to the posterior rib.

6. Bedside Preparation and Division of Posterior Rib (Video 6). The robot arms are decoupled from the anterior and posterior working ports and dismissed temporarily from the surgical field. The posterior port is removed, and the drill is inserted into the chest directly through the incision. A suction irrigator is placed through the anterior port. The surgeon gingerly divides

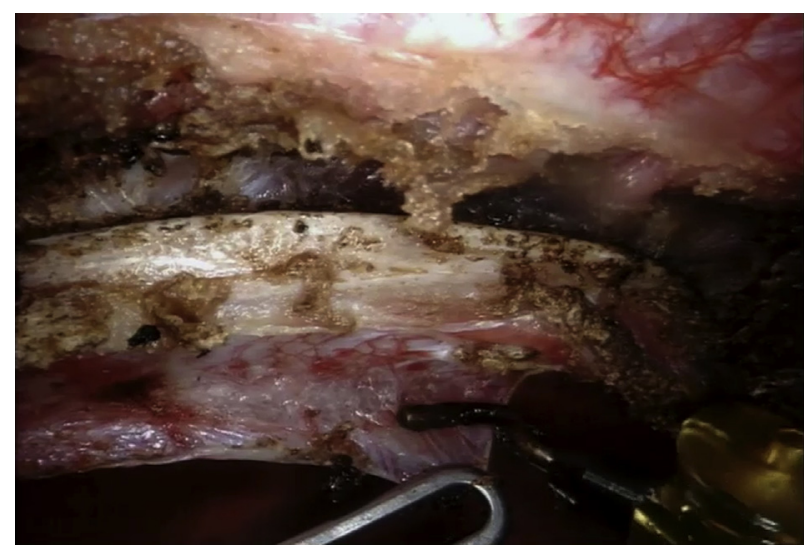

VIDEO 4. Dissection of mediastinal pleura from inferior aspect of first rib. Video available at: https://www.jtcvs.org/article/S2666-2507(20) 30017-1/fulltext. 


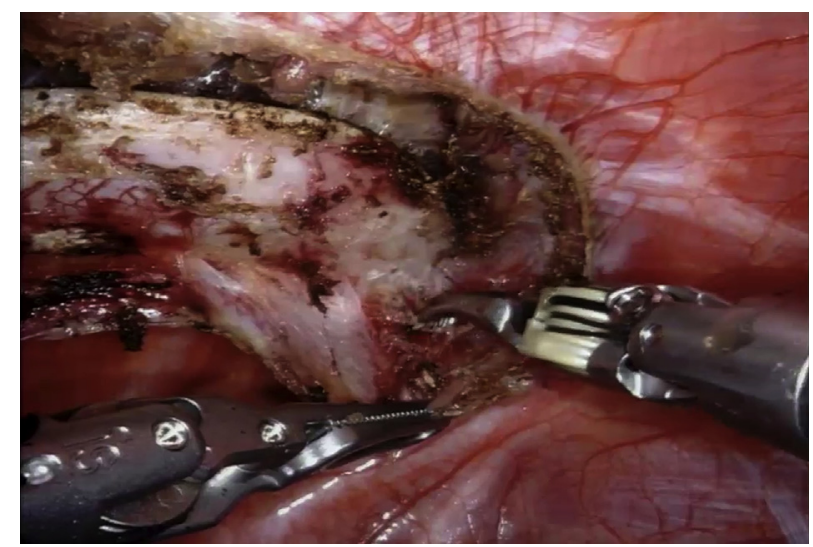

VIDEO 5. Division of costal cartilage and preparation of posterior rib. Video available at: https://www.jtcvs.org/article/S2666-2507(20)30017-1/ fulltext.

the posterior first rib with the drill while the assistant occasionally dribbles saline on the site of division. In cases of neurogenic TOS, it is believed to be important to divide the rib at a level approaching the transverse process. To mitigate any slipping of the drill, the surgeon can stabilize its shaft with her or his other hand and may activate the drill before its contact with the rib.

7. Division of Serratus Anterior and Dissection Along the Superior Rib (Video 7). The robot is re-docked, and the surgeon returns to the console. The first rib is now free from its anterior and posterior fixation and remains attached to the serratus anterior muscle, the middle and anterior scalene muscles, and the subclavius muscle and costoclavicular ligament. The rib is reflected medially into the thorax using the robotic pro-grasp instrument, and the serratus muscle is separated from the superior aspect of the first rib by dividing its attachments to the rib. The loose fascial attachments of the thoracic outlet can be gently swept

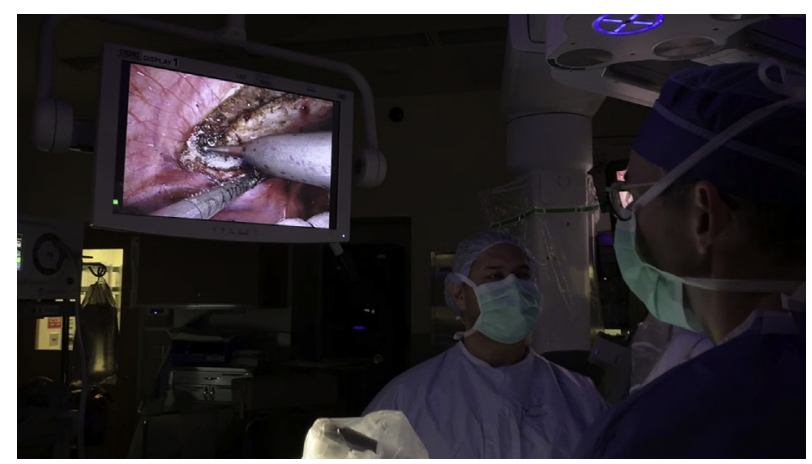

VIDEO 6. Bedside preparation and division of posterior rib. Video available at: https://www.jtcvs.org/article/S2666-2507(20)30017-1/fulltext.

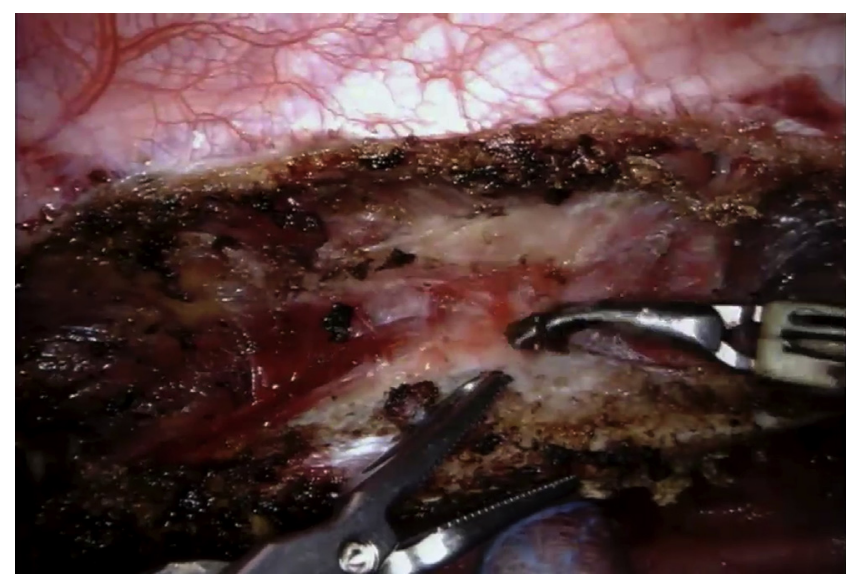

VIDEO 7. Division of serratus anterior and dissection along the superior rib. Video available at: https://www.jtcvs.org/article/S2666-2507(20) 30017-1/fulltext.

off of the rib, exposing the middle scalene muscle posteriorly, the subclavius muscle and costoclavicular ligament anteriorly, and the anterior scalene muscle in the middle portion of the rib.

8. Division of Middle Scalene Muscle (Video 8). Bundles of middle scalene muscle are divided at their attachments on the first rib. The T1 nerve root should be identified and protected during this step. Should the surgeon desire, a partial middle scalenectomy can be performed and may be simplest after the rib is fully detached.

9. Division of Subclavius Muscle and Costoclavicular Ligament (Video 9). The rib is reflected anteriorly, and the subclavius muscle is divided where it inserts on the rib. The costoclavicular ligament is divided in a similar fashion. These 2 structures can be afflicted by remarkable fibrosis in patients with vTOS but can often be easily "scraped" off of the rib. Because the

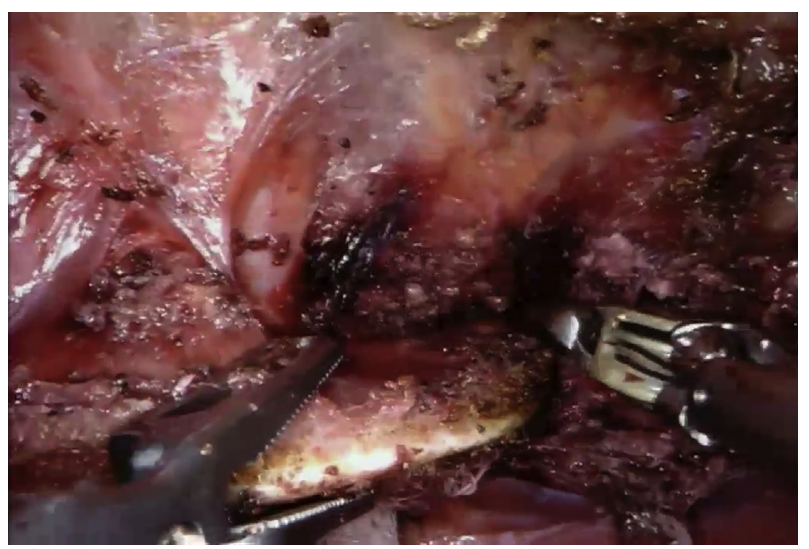

VIDEO 8. Division of middle scalene muscle. Video available at: https:// www.jtcvs.org/article/S2666-2507(20)30017-1/fulltext. 


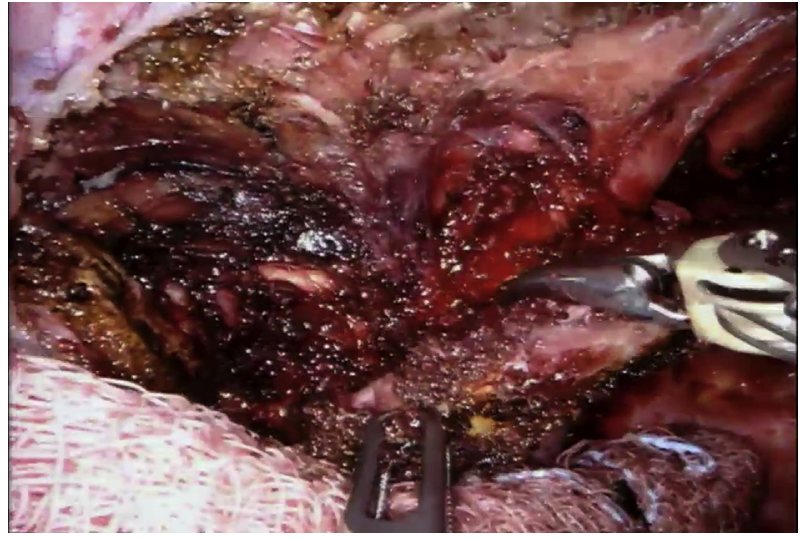

VIDEO 9. Division of subclavius muscle and costoclavicular ligament. Video available at: https://www.jtcvs.org/article/S2666-2507(20)30017-1/ fulltext.

soft tissue attachments posteriorly (the middle scalene muscle) and anteriorly (the subclavius muscle and costoclavicular ligament) can challenge the surgeon to achieve the desired medial rib reflection, this can be overcome by sequential, alternating progress on steps 8 and 9 .

10. Division of Anterior Scalene Muscle (Video 10). At this point, the rib is joined to the patient only by the anterior scalene muscle, which can be put on gentle stretch by retracting the rib. The location of the phrenic nerve will be known and typically emerges from the neck at a location deep (medial) to the first rib, but this route can be variable and an accessory phrenic nerve can lie closer to the medial aspect of the first rib. Scalene muscle fiber bundles are dissected and divided at their intersection with the first rib. The rib is released and temporarily placed to the side. If scalenectomy is desired, $2 \mathrm{~cm}$ or more of the middle and anterior scalene muscles can be resected by gentle

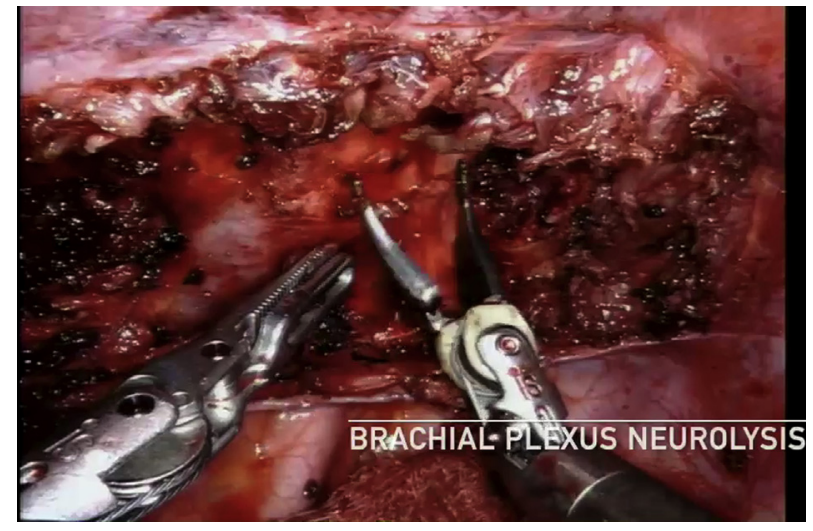

VIDEO 11. External venolysis or brachial plexus neurolysis. Video available at: https://www.jtcvs.org/article/S2666-2507(20)30017-1/fulltext.

retraction and resection. The authors prefer sharp division with robotic shears for which bleeding is selflimited.

11. External Venolysis or Brachial Plexus Neurolysis (Video 11). In cases of vTOS, it is common to find the subclavian vein buried in layers of fibrotic tissue, and an external venolysis should be considered. This can be performed in a variety of ways; however, energy should probably be limited, and thoracic surgeons inexperienced in neurolysis may choose to collaborate with their neurosurgeon colleagues. The curved bipolar dissector is a sharp-tipped instrument and is well suited for this step. The extent of external venolysis in vTOS, via any approach, has not been scientifically studied. The authors pursue a near circumferential dissection of the subclavian vein, 3 to $4 \mathrm{~cm}$ in length, to liberate the vein. In cases of neurogenic TOS, brachial plexus neurolysis is often performed by TOS surgeons immediately after FRR. If desired, the surgeon can complete a thorough brachial plexus neurolysis using

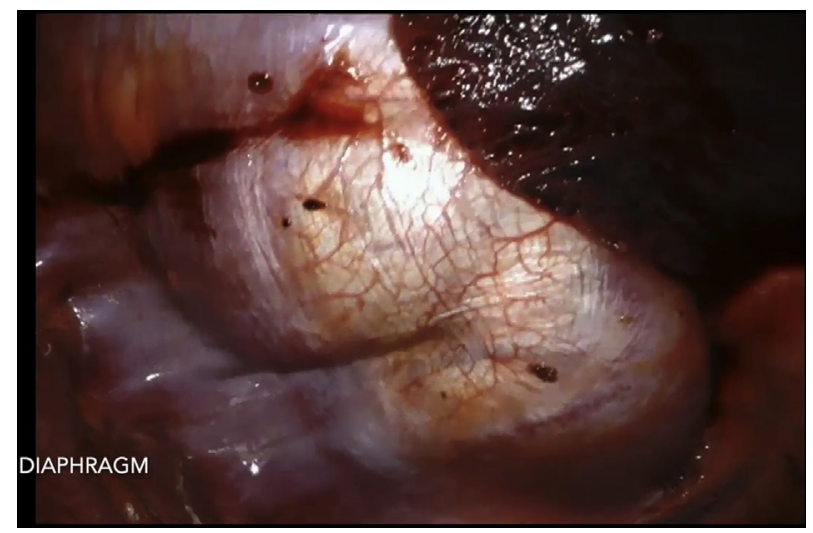

VIDEO 12. Removal of specimen. Video available at: https://www.jtcvs org/article/S2666-2507(20)30017-1/fulltext.
VIDEO 10. Division of anterior scalene muscle. Video available at: https://www.jtcvs.org/article/S2666-2507(20)30017-1/fulltext. 
similar sharp, nonenergy, dissection techniques. The author pursues a near-circumferential dissection of each trunk of the brachial plexus.

12. Removal of Specimen (Video 12). The assistant port is removed, and a long ring forceps (double action, if available) is used to remove the rib, which almost always can be extracted without enlarging the incision. Hemostasis is assured. A 24F Blake drain is placed into the chest, and the lung is reexpanded. If the surgeon chooses, she or he can have the neuromuscular blockade reversed during the final steps of the procedure and can observe the diaphragm briskly contracting.

\section{DISCUSSION}

The diagnosis of TOS and decision to proceed with surgery are facilitated through evaluation by an experienced multidisciplinary team. ${ }^{3}$ The predominant advantage of the transthoracic minimally invasive approach to resection of the first rib is its consistent, outstanding visualization of the first rib that is independent of body habitus. Transthoracic minimally invasive approaches to FRR have been reported and include the thoracoscopic-assisted transaxillary approach, ${ }^{4}$ pure thoracoscopic approaches, ${ }^{5}$ and transthoracic robotic approaches. ${ }^{6}$ Our specific approach for rFRR was derived from the technique described by Srother and Margolis in 2015. ${ }^{6}$ These transthoracic minimally invasive approaches are unique because they display to the surgeon the near entirety of the first rib immediately after beginning the operation.

In contrast to traditional approaches to FRR, rFRR does not require any retraction or manipulation of neurovascular structures. Although this is hypothesized to decrease the possibility of catastrophic intraoperative complications and surgical morbidity in the form of decreased brachial plexus injuries resulting in any sensory or motor deficit, decreased phrenic nerve injury, decreased bleeding events, and decreased thoracic duct injury, its comparative efficacy to other approaches has not been rigorously evaluated. In the largest published series of rFRR, however, Gharagozloo and colleagues ${ }^{7}$ reported outstanding short-term outcomes including no neurovascular complications in a cohort of 83 patients undergoing rFRR for vTOS. Specific techniques used to mitigate the chance of neurovascular injury during rFFR include beginning each case with a thorough survey of anatomy and identification of all neurovascular structures, exclusive use of bipolar energy during dissection around the medial aspect of the first rib, use of only sharp dissection (no energy) during brachial plexus neurolysis, and division of the rib far anteriorly and far posteriorly, which provides distance from subclavian artery, subclavian vein, phrenic nerve, and brachial plexus aside from the T1 nerve root that can be clearly visualized and protected during this step.

The intraoperative strategy to address major bleeding during rFRR is commonly and appropriately inquired on. Several management strategies are available to the surgeon in the case of this rare event, and all begin with holding pressure on the bleeding source using a robotic instrument held sponge. The exposure provided by this approach and the nimble precision provided by the robotic platform facilitate suture closure of a potential arteriotomy or venotomy. Such repair can be aided by the use of small "bulldog" clamps placed into the chest through the assistant port and applied using the robotic instrument (Video 11). Other possibilities to quell major arterial or venous bleeding include percutaneously placed balloon catheters or stents via the ipsilateral arm, which is accessible in the decubitus position. Finally, should it be required, an extended posterolateral thoracotomy while the assistant holds pressure on the bleeding source through the assistant port can provide open access to the thoracic outlet.

\section{CONCLUSIONS}

The 12 steps of this operation outlined in this report represent one group's approach to this procedure and should be further innovated.

\section{References}

1. Rinehardt EK, Scarborough JE, Bennett KM. Current practice of thoracic outlet decompression surgery in the United States. J Vasc Surg. 2017;66: 858-65.

2. Loukas M, Kinsella CR Jr, Louis RG Jr, Gandhi S, Curry B. Surgical anatomy of the accessory phrenic nerve. Ann Thorac Surg. 2006;82:1870-5.

3. Burt BM. Thoracic outlet syndrome for thoracic surgeons. J Thorac Cardiovasc Surg. 2018;156:1318-23.

4. Soukiasian HJ, Shouhed D, Serna-Gallgos D, McKenna R III, Bairamian VJ, McKenna RJ Jr. A video-assisted thoracoscopic approach to transaxillary first rib resection. Innovations (Phila). 2015;10:21-6.

5. George RS, Milton R, Chaudhuri N, Kefaloyannis E, Papagiannopoulos K. Totally endoscopic (VATS) first rib resection for thoracic outlet syndrome. Ann Thorac Surg. 2017;103:241-5.

6. Srother E, Margolis M. Robotic first rib resection. Oper Techn Thorac Cardiovasc Surg. 2015;20:176-88

7. Gharagozloo F, Meyer M, Tempesta B, Gruessner S. Robotic transthoracic first-rib resection for Paget-Schroetter syndrome. Eur J Cardiothorac Surg. 2019;55: 434-9. 